inhibitory stimulus control by $\mathrm{CS}-$, which could be "turned on and off" during consecutive minutes of the same conditioning trial.

\section{REFERENCES}

HAMMOND, L. J. Increased responding to $\mathrm{CS}^{-}$ in differential CER. Psychonomic Science, 1966, 51, 337-338.

HAMMOND, L. J. A traditional demonstration of the active properties of Pavlovian inhibition using differential CER. Psychonomic Science, 1967, 9, 65-66.

MILLER, L. Compounding of pre-aversive stimuli. Journal of the Experimental Analysis of Behavior, 1969, 12, 293-299.

REBERG, D., \& BLACK, A. H. Compound testing of individually conditioned stimuli as an index of excitatory and inhibitory properties. Psychonomic Science, 1969, 17 30-31.

RESCORLA, R. A., \& LOLORDO, V. M. Inhibition of avoidance behavior. Journal of Comparative \& Physiological Psychology, $1965,59,406-412$.

RESCORLA, R. A., \& SOLOMON, R. L. Two-process learning theory: Relationships between Pavlovian conditioning and instrumental learning. Psychological Review, $1965,74,151-182$.

\section{NOTES}

1. The total number of responses made when CS- appeared in Minute 1 were 33,128, 29, and 240 for Rats C21, C22, C23, and C24, respectively; when CS- appeared in Minute 2, the corresponding response totals were 69,41 , 34 , and 120.

\title{
Effect of competition on escape from noxious stimulation
}

LOUIS W. SUTKER, LAWRENCE GUBLER, JR., and C. J. WALLACE, Loyola University of the South, New Orleans, La. 70118

Twenty 90-day-old male Sprague-Dawley rats were separated into five homogeneous groups of four rats each on the basis of running speed to escape electrical shock in a runway. Within each of these groups, two $S s$ were assigned randomly to a competition condition and two to a noncompetition condition. All Ss were run in pairs in a double-alley runway under shock-escape conditions. The first animal in the competition pair to reach the goal was allowed to enter the goal and escape the shock. Ss in the noncompetition pairs were yoked to the competition pairs, and their reinforcement was contingent upon the performance of their yoked partners. In all cases, the performance of the competition Ss exceeded that of the noncompetition $S s \quad(p<.01)$, demonstrating the motivating effect of stimuli associated with the competitive situation.

Bayroff (1940) has demonstrated a competitive effect (increase in performance due to being allowed reinforcement in the presence of a nonreinforced $S$ ) in rats swimming in an underwater maze. The first rat to reach the goalbox was rewarded by immediate access to air, while the losing rat was deprived for an additional $20 \mathrm{sec}$. Of the total of $56 \mathrm{Ss}$ (28 pairs) $80.4 \%$ swam significantly faster under competition than under noncompetitive conditions.

Church (1962) has pointed out the methodological deficiencies of Bayroff's (1940) study. He has suggested that the latter's results were based on experimentation in which there was no control group to determine whether the increase in performance was due to such factors as (1) the additional number of trials, (2) the delay of reinforcement, or (3) the competitive allotment of reinforcement. Taking these factors into consideration, Church (1962) studied the effect of a competitive situation on lever pressing by albino rats in adjacent cages. On a 30-sec VI schedule, a food reinforcement was made available to both rats, and the first of the two experimental Ss to make the appropriate response received the pellet. These two experimental Ss were matched to a control pair rewarded not on the basis of their own response, but that of the experimental pair. Ss in the former group displayed a considerably greater increase in response rate over those in the control, noncompetition group.

Kanak \& Davenport (1967) used the design of Church (1962) and modified it slightly in order that the motivational influences of the $S s$ were more readily observable. By using the yoked design, competition effects on albino rats in a double-alley runway were studied. Sixteen male albino rats were divided into four homogeneous blocks by rank ordering the mean speed scores of five trials on the 3rd day of training. In each group, one pair was assigned randomly as competitive and the other as noncompetitive. Reinforcement in the competition pair was based on arriving at the goalbox first and being allowed entrance to the food pellets within. In the noncompetition pair, reinforcement was based not on their performance in the runway, but upon that of their yoked competition partner. The results were significant at the .05 level, demonstrating a competition effect.

The present study employed the noxious stimulation of Bayroff's (1940) investigation with the methods and design of Church (1962) and Kanak \& Davenport (1967) to explore the competition effect in the albino rat in escaping electric shock in a double-alley runway.

\section{APPARATUS}

The apparatus consisted of a straight double-alley runway, 48 in. in length, with 8-in.-long start- and goalboxes making the total length $64 \mathrm{in}$. Total width of the runway was $12 \mathrm{in}$., $6 \mathrm{in}$. for each half. The runway was $8 \mathrm{in}$. high and was provided with a clear plastic cover. The doors of the start- and goalboxes and the partition between the two alleys were made of clear Plexiglas. The doors to the start- and goalboxes were of the guillotine type raised by means of a string attached to the top of the Plexiglas and run through a support immediately above. Four microswitches, attached individually to the doors, operated timers to record latency and running speed. The startbox and alley had a grid floor connected to a Grason-Stadler electromechanical scrambling mechanism that provided the noxious stimulation. Shock intensity was $.8 \mathrm{~mA}$. The entire runway, except for the Plexiglas portions and the grid floor, was constructed of $1 / 4$-in. plywood sprayed on the inside with flat black paint.

SUBJECTS AND PROCEDURE

The Ss were 20 male Sprague-Dawley rats, approximately 90 days old, housed in individual cages with ad lib food and water supply. All Ss were rank-ordered on the basis of running speed following a 2-day initial escape training period of 30 trials per day on $100 \%$ reinforcement. Five homogeneous groups of four rats each were formed by placing the fastest four Ss together, then the next fastest four, and so on, down to the slowest four Ss. Within each of these blocks, two Ss were assigned randomly to a competition pair and two to a yoked noncompetition pair. The same pairs of rats always ran together.

Reinforcement was defined as entrance into the goalbox, where no shock was present, and was given only to the first rat reaching the goal chamber in the competition pair and to the rat in the noncompetition pair yoked to the winning $S$ regardless of performance. The losing $S$ and his yoked noncompetition partner were unrewarded, i.e., deprived entrance into the goalbox and maintained under shock for an additional $3 \mathrm{sec}$ for that 


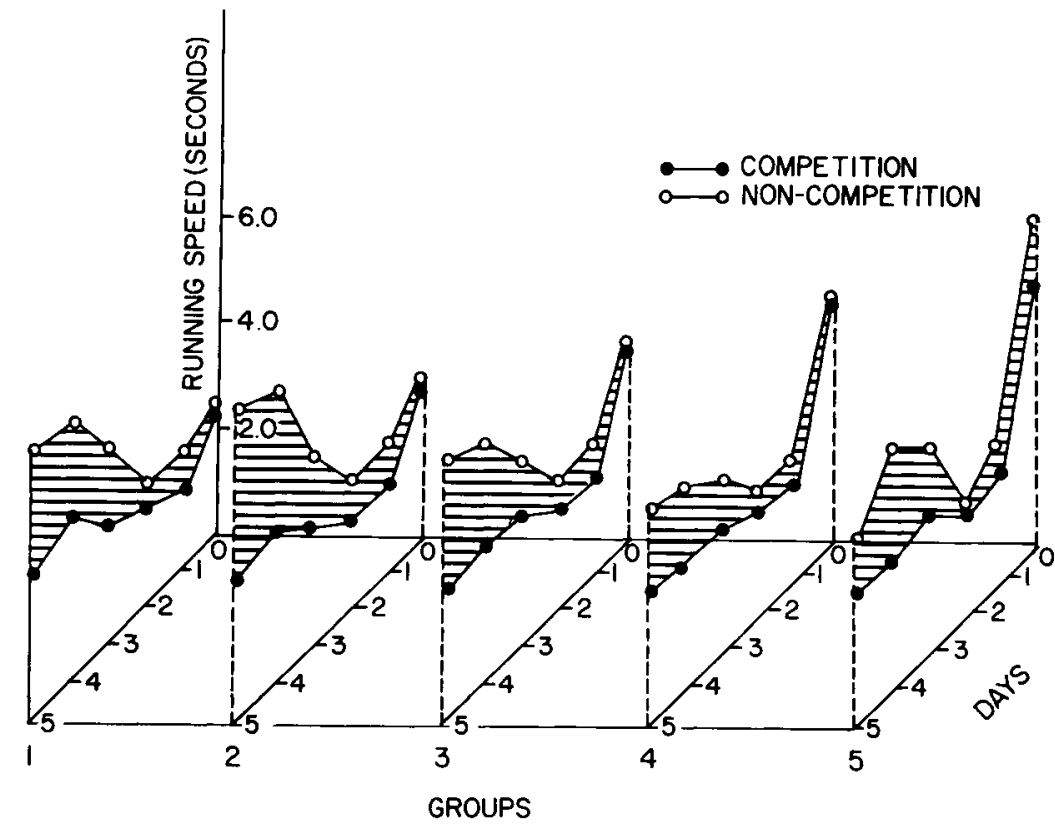

particular trial. All Ss were run 10 trials a day for 5 consecutive days.

\section{RESULTS AND DISCUSSION}

The means of the last 10 training trials for each $S$ were used as the dependent variable in a 5 by 2 factorial analysis of the training trials. There was a significant group effect $(F=11.87, \quad \mathrm{df}=4 / 10$, $\mathrm{p}<.01$ ), and a consistent decrease in running speed from the slowest to fastest groups. This was the effect sought at the outset of the ranking procedure. The second factor, competition vs noncompetition, was nonsignificant $(\mathrm{F}=.766, \mathrm{df}=1 / 10, \mathrm{p}>.05)$, indicating that both groups were approximately equal in running speed prior to testing. The interaction effect was also nonsignificant $(F=.512, \mathrm{df}=4 / 10, \mathrm{p}>.05)$.

The data for the test trials were analyzed by a 5 by 2 by 5 factorial design with repeated measures on the last factor (days).

The factor of key interest to this study, competition vs noncompetition was significant $(F=155.02, \quad \mathrm{df}=1 / 10$, $\mathrm{p}<.01$ ), and Fig. 1 shows that increased performance was found in the competition animals in all five groups over each of the 5 days. The competition Ss consistently reached the goal chamber faster than their yoked noncompetition partners. There was a significant group effect $(F=44.9$, $\mathrm{df}=4 / 10, \mathrm{p}<.01)$ reflecting continued differences in running speed between the five groups.
The third factor, days, was also significant $(F=8.36, \mathrm{df}=4 / 40, \mathrm{p}<.01)$. There was a general increase in running Competition by Group by Days interaction was not statistically significant $(F=1.45$, $\mathrm{df}=16 / 40, \mathrm{p}>.05)$.

This study and those conducted by Bayroff (1940), Church (1961, 1962), and Kanak \& Davenport (1967) support the evidence that, following the given procedures of a matched pair, yoked design, albino rats can be shown to exhibit increased performance in terms of running speed when placed in a competition speed from the 1 st to the 5 th day over all groups. The Competition by Days interaction effect was similarly significant $(F=4.18, \quad d f=4 / 40, \quad p<.01)$, and demonstrated the differential effects of additional trials on the two groups. Examination of Fig. 1 reveals that the performance of the competition group was more stable than that of the noncompetition group over the 5-day period. This can be attributed to the fact that the reward-punishment criterion was offered the competition pairs and not the noncompetition Ss. The noncompetition Ss were learning that reinforcement was

The Group by Days interaction was significant $(F=3.91, \mathrm{df}=16 / 40, \mathrm{p}<.01)$. An examination of the data and inspection of Fig. 1 show that the slowest two groups increased speed more over the 5-day period independent of running speed.
Fig. 1. Three-factor interaction showing the mean running speed of the competition and noncompetition $S s$ from the fastest (Group 1) to the slowest (Group 5) over days. Day 0 represents the mean of the last 10 trials for the last training day.

than did the fastest two groups. This may be attributed to greater punishment in terms of longer initial exposure to shock serving to intensify the motivation of these slower groups whose performance was nonasymptotic. It is to be noted that the poor performance of the slow groups during the training trials was determined by motivational and not ability, or learning, factors. Initially, both groups showed increased performance in the competitive situation, although there was a constant difference in favor of the competition group.

Competition by Group interaction was also significant $(F=9.46, d f=4 / 10$, $\mathrm{p}<.01)$ and due to the greater decrease in running speed of the slower groups under the noncompetition condition than under the competition condition. The situation in which escape from noxious stimulation is dependent upon relative speed in a straight-alley runway.

Carnathan \& Church (1964) have demonstrated that the competition effect, using appetitive drive, is not dependent on the actual presence of a competing $S$ but on the temporal contingency relationships involved. Further experimentation should determine if this finding could be extended to shock-motivated competition.

\section{REFERENCES}

BAYROFF, A. B. The experimental social behavior of animals: II. The effect of early isolation of white rats on their competition in swimming. Journal of Comparative \& Physiological Psychology, 1940, 29, 293-306. CARNATHAN, J., \& CHURCH, R. M. The effect of competitive allocation of reinforcement to rats in the straight alley. The Journal of General Psychology, 1964, 71, 137-144.

CHURCH, R. M. Effects of a competitive situation on speed of response. Journal of Comparative \& Physiological Psychology, 1961, 54, 162-166.

CHURCH, R. M. Effect of relative skill on the amount of competitive facilitation. Psychological Reports, 1962, 11, 603-614.

KANAK, N. J., \& DAVENPORT, D. G. Between-subject competition: A rat race. Psychonomic Science, 1967, 7, 87-88. 